\title{
A usability gap between older adults and younger adults on interface design of an Internet-based telemedicine system
}

\author{
Young J. Chun ${ }^{\mathrm{a}^{*}}$ and Patrick E. Patterson ${ }^{\mathrm{a}}$ \\ ${ }^{a}$ Department of Industrial Engineering, Texas Tech University, 2500 Broadway, Lubbock, TX 79409, USA
}

\begin{abstract}
Internet-based telemedicine is becoming an effective tool to deliver home-healthcare services and health information on demand, especially in rural areas, where there is often a large elderly population with greater rates of preventable chronic diseases. However, the designs of current interfaces for these internet-based telemedicine systems do not take elderly user characteristics into consideration. This study conducted usability testing on the interface of an internet-based telemedicine system using two different age groups, young adults and older adults. Differences in overall performance and satisfaction between the two groups were identified. Based on these results, a future direction is suggested for the interfaces of internetbased telemedicine systems.
\end{abstract}

Keywords: internet-based telemedicine system, usability, interface design, older adults, elderly users

\section{Introduction}

Internet-based telemedicine systems have become an effective method for delivering home-healthcare services and health-related information, especially in rural areas which tend to have larger elderly populations with higher chronic disease rates than urban areas $[5,7]$.

The number of the elderly keeps increasing, with $20 \%-22 \%$ of the US population is expected to be older than 65 years by 2030 [1,6]. Meanwhile, chronic disease exponentially increases with aging [8]. Since the elderly with chronic diseases require constant self-care as well as care provided by professionals, timely health information provided through an internet-based telemedicine system is even more critical for them [4].

The elderly obviously have differences in physical and cognitive capabilities from younger adults [3]. These limited capabilities may interfere with the elderly's use of web interfaces in telemedicine systems. However, there has been very little research that examines the interfaces of current internet-based telemedicine systems from an HCI perspective for elderly users.

This study conducted usability testing on the interface of an internet-based telemedicine system "My HealtheVet." The objective of the study is to identify the difference in performance levels and satisfaction between the younger and older adult groups, and identify the interface factors that cause those differences.

\section{Methods}

\subsection{Participants}

A total of sixteen people, ten younger adults and six older adults participated in this study. The average age of the younger group was 27.6 years old $(\mathrm{SD}=3.10$, range between 24 and 33 years old $)$ and that of the older adult group was 61.3 years old (SD $=6.95$, range between 54 and 73 years old), respectively. The younger group had seven male and three female participants and the older adult group

*Corresponding author. E-mail: youngji.chun@ttu.edu. Tel: 1-806-742-3543 (Department of Industrial Engineering). 
had three male and three female subjects. All participants had web browsing experience and had basic internet skills. Each younger adult participant considered their computer skills to be average and above, while two of the older adult group evaluated as novices. None of the participant had any disability that would affect the experiment results.

\subsection{Measures}

Four primary measures were chosen: task completion rate (effectiveness), task completion time (efficiency), the number of errors (errors), and satisfaction (subjective preference). The number of errors was defined as the number of wrong web pages visited, deviations from the optimal pathways.

\subsection{Apparatus}

The apparatus consisted of two Dell Precision 390 desk top computers, two Dell L04 REV.C1 LCD screens, Window XP Operating System, Internet Explorer Browser, and Shared T1 connection. A consent form, a demographic form, task scenarios, moderator packages, Lewis' after-task questionnaire, and Lewis' post-study questionnaire were also prepared [2].

\subsection{Tasks}

One practice task and eight actual tasks were given. These tasks were chosen based on task analysis considering frequency and essentiality. The tasks were also designed to agree with the purpose of telemedicine system. To minimize any confounding effect of order, task order was randomized for every participant. The task descriptions are as follows.

- Practice trial task 1. Log-in to the website

- Task 1. Find a clinic facility near me

- Task 2. Add your allergy history

- Task 3. Find the phone number of your health care provider (physician)

- Task 4. Find your previous blood pressure

- Task 5. View your previous medications information

- Task 6. Find information for healthy eating

- Task 7. Find common symptoms of Diabetes Type 2

- Task 8. Launch the 24hr health information radio about National Institute of Health (NIH)

\subsection{Procedure}

When the participants arrived, the objective of the study and the procedure were explained. Then, the consent form and demographic form were filled out. After adjusting their sitting position, task descriptions were given. The participants then performed given tasks with a 200 seconds time limit for each task. After completing a task, each participant filled out an after-task questionnaire which was related to the corresponding task. After finishing all eight tasks, the subjects were asked to express their feelings about the overall website through a post-study questionnaire. After all testing was completed a posttest interview was conducted to obtain their opinions about the web interface of the internet-based telemedicine system.

\section{Results}

\subsection{Task completion rate}

Overall task completion rate (success of task performance) across all eight tasks of the younger group was $80.0 \%$ while the older adult group had a $64.6 \%$ task completion rate. Even though not statistically significant ( $\mathrm{p}$-value $=0.073$ ), there seemed to be an obvious tendency for the younger adult users to perform more successfully than the older adult users. In particular, there were great differences in task $1(90.0 \%$ vs. 50.0\%) and task 2 $(90.0 \%$ vs. $33.3 \%)$ between the two age groups. And both groups showed relatively low task completion rates on task $7(70.0 \%$ vs. $50.0 \%)$ and task $8(30.0 \%$ vs. $16.7 \%$ ). while both groups showed comparatively high task completion rate for task $3(100.0 \%$ vs. $100.0 \%)$, task $4(100.0 \%$ vs. $90.0 \%)$, and task 6 (90.0\% vs. $100.0 \%)$.

\subsection{Task completion time}

There was a significant difference in task completion time between two age groups ( $p$-value 0.048). Overall task completion time across all eight tasks was 71.85 seconds for the younger group and 80.11 seconds for the older adult group. The younger adult users especially showed a much shorter time than the older adult group did for Task 1 (69.3 sec vs. $138.0 \mathrm{sec})$, task $2(90.9 \mathrm{sec}$ vs. $116.0 \mathrm{sec})$, task 5 $(62.4 \mathrm{sec}$ vs. $91.3 \mathrm{sec})$, task $6(71.2 \mathrm{sec}$ vs. $84.8 \mathrm{sec})$, and task 7 (91.7 sec vs. $114.0 \mathrm{sec})$. Only one older 
subject succeeded on task 8, but only used 52.0 seconds while the younger group averaged 143.0 seconds.

\subsection{The number of errors}

The difference in the number of errors between two age groups was statistically significant ( $\mathrm{p}$-value $=0.003$ ). Overall average number of errors for the younger adult group was 3.59, and that of the older adult group was 2.55 , respectively.

The younger adults group had the higher error rate, a result that may be due to the younger adult tendency to have faster information processing and decision making process than did the older adult group when using the interface. The older adults were more likely to stay longer on one web page and not proceed to the next page until they were quite sure about their paths.

\subsection{Satisfaction}

The after-task questionnaire contains three questions about satisfaction dealing with the ease, amount of time, and support information for completing the assigned tasks. There was a sevenpoint rating scale on this questionnaire, where a score of ' 1 ' indicates strong satisfaction and a score of '7' indicates strong dissatisfaction.

The overall average rating of satisfaction for the ease was $3.41(\mathrm{SD}=2.27)$ and 3.54 , for the amount of time was $3.46(\mathrm{SD}=2.33$ ) and 3.56 , and for support information $3.55(\mathrm{SD}=2.00)$ and 3.46 in the younger group and in the older adult group, respectively, even though these was no statistically significant difference between two age groups ( $\mathrm{p}$ value $=0.949$ ). However, there was a pattern of the older adult group which showed relatively higher dissatisfaction in task 2 with rating range between 5.67 and 6.10 and both groups showing higher dissatisfaction with rating between 4.83 and 5.17 in task 8.

The post-study questionnaire included 21 multiple choice questions and two write-out questions. The younger adults group showed dissatisfaction about the web interface for 15 questions out of a total of 21 questions. The same scale used for the after-task questionnaire was also used for the post-study questionnaire.

The overall average rating was 4.10, slightly toward dissatisfaction in the younger group and 3.58, an about average rating in the older adult group.
Even though these was no significant statistical difference $(p$-value $=0.603$ ) between two groups, the younger group showed relatively high dissatisfaction (range points above 4.5) for five question while the older adult group had only one question with high dissatisfaction of 4.67 .

When compared to the younger adult group, the older adult group tended to be more highly, and easily satisfied, once they had successfully completed the tasks.

\subsection{Significant problems and interface factors}

Both younger and older adult groups were satisfied with the broad ranges of health-related and healthcare functions provided by the internet-based telemedicine system. These functions allow users to take various actions about their health issues, such as to get health care from their physicians, to keep track of their health history, to get research based health related information, and so forth in real time.

However, both groups particularly complained of the menu organization and structure. First of all, the menus lacked a hierarchical structure. Main menus and submenus were not properly linked each other. The menu contents did not match with users' mental models when they search the location of the particular information they needed. Second, menu content was cluttered and unclear. Because of this, users were not able to intuitively find the proper pathways to the intended information.

In addition, both groups had confusion with the menu bars due to the several different menu layers located vertically and horizontally. There were three horizontal menu bars on the top and two vertical menu bars on the right side of the homepage. Both the younger adult group and the older adult group could not determine which menu bar they needed to focus on as their first priority.

One of the other major issues mentioned by both user groups was the poor help function and limited search capability. When they found themselves stuck while using the interface, the help function and the search function did not provide supportive information to aid the users in recovering from the errors.

Another issue was legibility. In particular, the older adult group had a difficult time due to the bland graphics, poor color contrast, small letter size, and thin letters in the menu bar. Since elderly populations obviously have limited visual capabilities as compared to younger people, older adults are more 
sensitive about problems that might negatively affect their reading and performance. Overall, the content on each of the menu bars was not salient enough to catch user's attention instantly.

\section{Conclusion and discussion}

When there is no direct and obvious cue on the homepage, the older adults tended to have more difficulty inferring where to click to proceed to the next step. They spent more time considering where to go for accessing the proper content.

Furthermore, one interesting thing found was that the older adults tended to blame themselves rather than the interface features when they could not complete the tasks more often than the younger adults did. This might be because older adults are less confident about using the internet, especially an internet-based telemedicine system that they do not use on a regular basis- even though they already know how to use the internet and have web browsing experience.

One of the biggest future challenges for interface design for internet-based telemedicine systems for elderly users is to provide proper organization and structure for the massive amount of health related information. The older adult group was more likely to have hard time performing the tasks associated with finding medical research information, especially about health and diseases. One older adult with a medical background performed better than the other older adult group participants. A dictionary or glossary for medical and health terms on a homepage would also be helpful for users not familiar with medical terminology. Once again, since information about health and diseases covers a very wide scope, a well organized menu structure, which matches to user mental models, is absolutely required. Concisely and clearly labeled contents are also important with regard to medical and health information in order to help older adults use an internet-based telemedicine system more effectively, efficiently, and easily.

\section{References}

[1] B. H. Beith, Needs and requirements in health care for the older adults: Challenges and opportunities for the new millennium, in: Human Factors Interventions for the Health Care of Older Adults, W. A. Rogers and A. D. Fisk, eds., Lawrence Erlbaum Associates, Inc., New Jersey, 2001, pp. 13-30.

[2] C. Lewis, P. Polson, C. Wharton and J. Rieman, Testing a walkthrough methodology for theory-based design of walk-upand-use interfaces, in: Proceedings from CHI '90: Human Factors in Computing Systems Conference. April, 1990, pp. 235-242.

[3] H. W. Wahl and C. Tesch-Römer, Aging, sensory loss, and social functioning, in: Communication, Technology, and Aging: Opportunities and Challenges for the Future, N. Charness, D. C. Parks and B. A. Sabel, eds., Springer, New York, 2001, pp. 108-126

[4] J. A. Loera, Generational differences in acceptance of technology, Telemedicine and e-Health 14(10) (2008), 10871090

[5] J. Lorca, F. J. Alcazar, C. L. Sanchez, M. Romero-Cuevas, D. M. Lopez, S. Ruiz and P. Garcia-Fortea, PESCA: Open source platform for eHealth, in: eHealth: Combining Health Telematics, Telemedicine Biomedical Engineering and Bioinformatics to the Edge: CeHR Conference Proceedings 2007, B. Blobel, P. Pharow, J. Zvarova and D. Lopez, eds., IOS Press, Berlin, 2008, pp. 65-70.

[6] J. M. Machibroda, Engaging consumers in health care advocacy using the internet, in: Health communication in the new media landscape, J. C. Parker and E. Thorson, eds., Springer Publishing Company, LLC, New York, 2008, pp. 267-282.

[7] P. S. Seibert, T. A. Whitmore, C. M. Patterson, C. C. Otto, P. D. Parker, N. Whitener, M. J. Ward, J, Basom and C. G. Zimmerman, Telemedicine research: Opportunities and challenges. in: Mobile Telemedicine: A Computing and Networking Perspective, Y. Xiao and H. Chen, eds., CRC Press, Florida, 2008, pp. 349-366

[8] R. L. Glueckauf and M. L. A. Lustria, E-health self-care interventions for persons with chronic illnesses: Review and future direction, in: Health communication in the new media landscape, J. C. Parker and E. Thorson, eds., Springer Publishing Company, LLC., New York, 2008, pp. 151-242. 